hep-th/0502146

QMUL-PH-05-02

\title{
A Recursion Relation for Gravity Amplitudes
}

\author{
James Bedford, Andreas Brandhuber, Bill Spence and Gabriele Travaglini * \\ Department of Physics \\ Queen Mary, University of London \\ Mile End Road, London, E1 $4 \mathrm{NS}$ \\ United Kingdom
}

\begin{abstract}
Britto, Cachazo and Feng have recently derived a recursion relation for tree-level scattering amplitudes in Yang-Mills. This relation has a bilinear structure inherited from factorisation on multi-particle poles of the scattering amplitudes - a rather generic feature of field theory. Motivated by this, we propose a new recursion relation for scattering amplitudes of gravitons at tree level. Using this, we derive a new general formula for the MHV tree-level scattering amplitude for $n$ gravitons. Finally, we comment on the existence of recursion relations in general field theories.
\end{abstract}

\& \{j.a.p.bedford, a.brandhuber, w.j.spence, g.travaglini $\} @ q m u l . a c . u k$ 


\section{Introduction}

Much progress has been made in the past year in understanding the structure and practical calculation of scattering amplitudes in four-dimensional Yang-Mills theories [1]. This has been prompted by the conjecture that twistor string theory provides a dual description of weakly-coupled gauge theory [2].

Outstanding progress in the efficient calculation of scattering amplitudes was achieved by Cachazo, Svrček and Witten (CSW) [3], who proposed a radical new method for computing tree-level amplitudes in Yang-Mills using MHV amplitudes, appropriately continued off shell, as vertices. This procedure was later extended to one loop in [4], and used to re-derive one-loop MHV scattering amplitudes in $\mathcal{N}=4$ super Yang-Mills.

Further progress in the calculation of one-loop amplitudes was achieved using the cutconstructibility approach [5,6] in [7], CSW diagrams [8-11], the holomorphic anomaly [1215] and generalized cuts [16-18]. An interesting spin-off of the latter approach was that the new results for loop amplitudes could be used to find new representations of tree-level amplitudes [17]. This is a direct consequence of the structure of infrared singularities of one-loop scattering amplitudes in gauge theory. Inspired by these insights, a new recursion formula for tree-level scattering amplitudes was proposed in [19], which is quadratic in the amplitudes and leads to very compact formulae. Recently, this proposal was proved in [20] using analyticity and factorization properties of gauge theory amplitudes. Recurrence relations were also derived in [21] to determine the rational part of one-loop amplitudes in QCD.

A key ingredient in the proof of the recursion relation in [20] is the fact that scattering amplitudes in Yang-Mills factorise on multi-particle poles. This is a fully non-perturbative statement and a general property of field theory. ${ }^{1}$ As such, it leads one to suspect that it should be possible to write down recursion relations for scattering amplitudes in generic theories which admit a field-theoretical description. In this paper we explore this idea, and propose a new recursion relation for the tree-level scattering of gravitons. The relation we prove is directly inspired by the $\mathrm{BCF} / \mathrm{BCFW}$ relation $[19,20]$, but takes into account the specific features which arise when considering gravity amplitudes.

One of the appealing features of the recursion relation of [19] is that it generates new formulae for amplitudes, which are often of a simpler form. We will show that the gravity recursion relation we propose also leads to a new formula for the $n$-point MHV amplitude for gravity scattering, which agrees with an earlier formula derived by Berends, Giele and Kuijf [23]. Guided by preliminary investigation of next-to-MHV gravity amplitudes, we expect that these recursion relations are also correct for more general tree-level gravity amplitudes.

\footnotetext{
${ }^{1}$ See, for example, chapter 10 of [22].
} 
The plan of the rest of the paper is as follows: In section 2, drawing inspiration from the BCFW proof [20] of the recursion relation for tree-level scattering amplitudes in YangMills, we derive a recursion relation for scattering amplitudes of gravitons at tree level. In section 3 we apply this recursion relation, and derive a new expression for the infinite sequence of MHV scattering amplitudes of gravitons. Finally, section 4 is devoted to some comments on recursion relations in other field theories.

For other recent work on gravity amplitudes see [24-27]. For related work on gauge theory amplitudes, see [29]-[40].

\section{The recursion relation in gravity}

In this section we closely follow the proof of the recursion relation in Yang-Mills [20], which we will extend to the case of gravity amplitudes. As we shall see, the main new ingredient is that gravity amplitudes depend on more kinematical invariants than the corresponding Yang-Mills amplitudes, namely those which are sums of non-cyclically adjacent momenta; hence, more multi-particle channels should be considered.

To derive a recursion relation for scattering amplitudes, we start by introducing a oneparameter family of scattering amplitudes, $\mathcal{M}(z)$ [20], where we choose $z$ in such a way that $\mathcal{M}(0)$ is the amplitude we wish to compute. We work in complexified Minkowski space and regard $\mathcal{M}(z)$ as a complex function of $z$ and the momenta. One can then consider the contour integral [21]

$$
\mathcal{C}_{\infty}:=\frac{1}{2 \pi i} \oint d z \frac{\mathcal{M}(z)}{z}
$$

where the integration is taken around the circle at infinity in the complex $z$ plane. Assuming that $\mathcal{M}(z)$ has only simple poles at $z=z_{i}$, the integration gives

$$
\mathcal{C}_{\infty}=\mathcal{M}(0)+\sum_{i} \frac{[\operatorname{Res} \mathcal{M}(z)]_{z=z_{i}}}{z_{i}}
$$

In the important case of Yang-Mills amplitudes, $\mathcal{M}(z) \rightarrow 0$ as $z \rightarrow \infty$, and hence $\mathcal{C}_{\infty}=0$.

Notice that up to this point the definition of the family of amplitudes $\mathcal{M}(z)$ has not been given - we have not even specified the theory whose scattering amplitudes we are computing.

There are some obvious requirements for $\mathcal{M}(z)$. The main point is to define $\mathcal{M}(z)$ in such a way that poles in $z$ correspond to multi-particle poles in the scattering amplitude $\mathcal{M}(0)$. If this occurs, then the corresponding residues can be computed from factorisation 
properties of scattering amplitudes (see, for example, $[22,28]$ ). In order to accomplish this, $\mathcal{M}(z)$ was defined in $[19,20]$ by shifting the momenta of two of the external particles in the original scattering amplitude. For this procedure to make sense, we have to make sure that even with these shifts overall momentum conservation is preserved, and that all particle momenta remain on-shell. We are thus led to define $\mathcal{M}(z)$ as the scattering amplitude $\mathcal{M}\left(p_{1}, \ldots, p_{k}(z), \ldots, p_{l}(z), \ldots, p_{n}\right)$, where the momenta of particles $k$ and $l$ are shifted to

$$
p_{k}(z):=p_{k}+z \eta, \quad p_{l}(z):=p_{l}-z \eta .
$$

Momentum conservation is then maintained. As in [19], we can solve $p_{k}^{2}(z)=p_{l}^{2}(z)=0$ by choosing $\eta=\lambda_{l} \tilde{\lambda}_{k}$ (or $\eta=\lambda_{k} \tilde{\lambda}_{l}$ ), which makes sense in complexified Minkowski space. Equivalently,

$$
\lambda_{k}(z):=\lambda_{k}+z \lambda_{l}, \quad \tilde{\lambda}_{l}(z):=\tilde{\lambda}_{l}-z \tilde{\lambda}_{k}
$$

with $\lambda_{l}$ and $\tilde{\lambda}_{k}$ unshifted.

More general families of scattering amplitudes can also be defined, as pointed out in [21]. For instance, one can single out three particles $k, l, m$, and define

$$
p_{k}(z):=p_{k}+z \eta_{k}, \quad p_{l}(z):=p_{l}+z \eta_{l}, \quad p_{m}(z):=p_{m}+z \eta_{m},
$$

where $\eta_{k}, \eta_{l}$ and $\eta_{m}$ are null and $\eta_{k}+\eta_{l}+\eta_{m}=0$. Imposing $p_{k}^{2}(z)=p_{l}^{2}(z)=p_{m}^{2}(z)=0$, one finds the solution

$$
\eta_{k}=-\alpha \lambda_{k} \tilde{\lambda}_{l}-\beta \lambda_{k} \tilde{\lambda}_{m}, \quad \eta_{l}=\alpha \lambda_{k} \tilde{\lambda}_{l}, \quad \eta_{m}=\beta \lambda_{k} \tilde{\lambda}_{m}
$$

for arbitrary $\alpha$ and $\beta$. This has been used in [21]. In the following we will limit ourselves to shifting only two momenta as in [19] and [20].

At tree level, scattering amplitudes in field theory can only have simple poles in multiparticle channels; for $\mathcal{M}(z)$, these generate poles in $z$ (unless the channel contains both particles $k$ and $l$, or none). Indeed, if $P(z)$ is a sum of momenta including $p_{l}(z)$ but not $p_{k}(z)$, then $P^{2}(z)=P^{2}-2 z(P \cdot \eta)$ vanishes at $z_{P}=P^{2} / 2(P \cdot \eta)[20]$. In Yang-Mills theory, one considers colour-ordered partial amplitudes, which have a fixed cyclic ordering of the external legs. This implies that a generic Yang-Mills partial amplitude can only depend on kinematical invariants made of sums of cyclically adjacent momenta. Hence, tree-level Yang-Mills amplitudes can only have poles in kinematical channels made of cyclically adjacent sums of momenta.

For gravity amplitudes this is not the case, as there is no such notion of ordering for the external legs. Therefore, the multi-particle poles which produce poles in $z$ are those obtained by forming all possible combinations of momenta which include $p_{k}(z)$ but not $p_{l}(z)$. This is the only modification to the BCFW recursion relation we need to make, in order to derive a gravity recursion relation. 
For any such multi-particle channel $P^{2}(z)$, we have

$$
\mathcal{M}(z) \rightarrow \sum_{h} \mathcal{M}_{L}^{h}\left(z_{P}\right) \frac{1}{P^{2}(z)} \mathcal{M}_{R}^{-h}\left(z_{P}\right),
$$

as $P^{2}(z) \rightarrow 0$ (or, equivalently, $z \rightarrow z_{P}$ ). The sum is over the possible helicity assignments on the two sides of the propagator which connects the two lower-point tree-level amplitudes $\mathcal{M}_{L}^{h}$ and $\mathcal{M}_{R}^{-h}$. It follows that

$$
[\operatorname{Res} \mathcal{M}(z)]_{z=z_{P}}=-\sum_{h} \mathcal{M}_{L}^{h}\left(z_{P}\right) \frac{z_{P}}{P^{2}} \mathcal{M}_{R}^{-h}\left(z_{P}\right)
$$

so that finally

$$
\mathcal{M}(0)=\mathcal{C}_{\infty}+\sum_{P, h} \frac{\mathcal{M}_{L}^{h}\left(z_{P}\right) \mathcal{M}_{R}^{-h}\left(z_{P}\right)}{P^{2}} .
$$

The sum is over all possible decompositions of momenta such that $p_{k} \in P$ but $p_{l} \notin P$.

If $\mathcal{C}_{\infty}=0$, then there is no boundary term in the recursion relation, and

$$
\mathcal{M}(0)=\sum_{P, h} \frac{\mathcal{M}_{L}^{h}\left(z_{P}\right) \mathcal{M}_{R}^{-h}\left(z_{P}\right)}{P^{2}} .
$$

In [20] it was shown that for Yang-Mills amplitudes boundary terms $\mathcal{C}_{\infty}^{\mathrm{YM}}$ always vanish. Two different proofs were presented, the first based on the use of CSW diagrams [3], the second on Feynman diagrams. For gravity, we still lack a description in terms of MHV vertices, so we can only rely on Feynman diagrams. This is also the case for other field theories we might be interested in (such as $\lambda \phi^{4}$, for example). As we have remarked, $\mathcal{C}_{\infty}=0$ if $\mathcal{M}(z) \rightarrow 0$ as $z \rightarrow \infty$. $\mathcal{M}(z)$ is a scattering amplitude with shifted, $z$-dependent external null momenta. One can then try to estimate the behaviour of $\mathcal{M}(z)$ for large $z$ by using power counting (different theories will of course give different results). In $\lambda \phi^{4}$ the Feynman vertices are momentum independent and $\mathcal{C}_{\infty}=0$ (see the last section); in quantum gravity, however, vertices are quadratic in momenta, and one cannot determine a priori whether or not a boundary term is present.

From the previous discussion, it follows that the behaviour of $\mathcal{M}(z)$ as $z \rightarrow \infty$ is related to the high-energy behaviour of the scattering amplitude (and hence to the renormalisability of the theory). The ultraviolet behaviour of quantum gravity, however, is full of surprises (for a summary, see for example section (2.2) of [41]). We may therefore expect a more benign behaviour of $\mathcal{M}(z)$ as $z \rightarrow \infty$. Specifically, in the next section we will focus on the MHV scattering amplitudes of $n$ gravitons, which have been computed by Berends, Giele and Kuijf (BGK) in [23]. Performing the shifts (2.3) explicitly in the BGK formula, one finds the surprising result ${ }^{2}$

$$
\lim _{z \rightarrow \infty} \mathcal{M}_{\mathrm{MHV}}(z)=0
$$

\footnotetext{
${ }^{2}$ We have checked that $\mathcal{M}(z) \sim \mathcal{O}\left(1 / z^{2}\right)$ as $z \rightarrow \infty$, analytically for $n \leq 7$ legs, and numerically for $n \leq 11$ legs.
} 
In more general amplitudes one can (at least in principle) use the (field theory limit of the) KLT relations [42], which connect tree-level gravity amplitudes to tree-level amplitudes in Yang-Mills, to estimate the large- $z$ behaviour of the scattering amplitude. ${ }^{3}$ As an example, we have considered the next-to-MHV gravity amplitude $\mathcal{M}\left(1^{-}, 2^{-}, 3^{-}, 4^{+}, 5^{+}, 6^{+}\right)$, and performed the shifts as in (2.4), with $k=1$ and $l=2$. Similarly to the MHV case, we find that

$$
\lim _{z \rightarrow \infty} \mathcal{M}\left(1^{-}, 2^{-}, 3^{-}, 4^{+}, 5^{+}, 6^{+}\right)(z)=0 .
$$

It would be interesting to understand if this is true for generic gravity amplitudes.

In the next section we will apply the recursion relation (2.10) to the case of MHV amplitudes in gravity, and show that it does generate correct expressions for the amplitudes. As a bonus, we will derive a new closed form expression for the $n$-particle scattering amplitude.

Before moving to the explicit computations, we would like to make some comments on how momentum shifts may be generated inside amplitudes. To begin with, we should notice that there is an intriguing difference between the MHV diagram method and the $\mathrm{BCF}$ recursion relation, namely the fact that in the latter one sums only over a subset of channels - those where the reference legs $k$ and $l$ are on different sides with respect to the internal propagator. It is clear that any derivation (rigorous or heuristic) of the recursion relation from the MHV diagrams method, or vice versa, will have to address this point. In the following we limit ourselves to some formal observations aimed at making a preliminary connection between the MHV diagram method and the shifts in the sub-amplitudes appearing in the recursion relation.

Let $\mathcal{A}$ be a tree-level scattering amplitudes of $n$ gluons in Yang-Mills, and let us focus on a particular channel $P_{i j}:=p_{i}+\cdots+p_{j}$. As $P_{i j}^{2} \rightarrow 0$, the scattering amplitude factorises as

$$
\mathcal{A} \rightarrow \sum_{h} \mathcal{A}_{L}^{h}\left(j+1, \ldots, i-1, P_{i j}\right) \frac{1}{P_{i j}^{2}} \mathcal{A}_{R}^{-h}\left(-P_{i j}, i, \ldots, j\right) .
$$

Now imagine that we want to construct the full amplitude - at $P_{i j}^{2} \neq 0$ - from MHV diagrams. The issue then arises of determining the spinors associated with the non-null momentum $P_{i j}^{2}$. A prescription equivalent to those of CSW consists of decomposing [33] ${ }^{4}$

$$
P=\lambda \tilde{\lambda}+z \eta
$$

where $\eta$ is a reference null momentum. $\lambda$ and $\tilde{\lambda}$ are then the spinors associated with the momentum $P$. The decomposition (2.14) played a central rôle in the calculation of one-loop amplitudes from MHV diagrams performed in $[4,9,11]$. Attached to the scalar propagator with momentum $P$ there will be two effective amplitudes $\mathcal{A}_{L}$ and $\mathcal{A}_{R}$ computed

\footnotetext{
${ }^{3}$ See the appendix for explicit examples of KLT relations for four, five and six legs.

${ }^{4}$ In the following we drop the subscript $i j$ in $P_{i j}$.
} 
from MHV diagrams. We will use $\lambda$ and $\tilde{\lambda}$ as spinors associated with $P$, and write the contribution to the amplitude as (schematically)

$$
\mathcal{A}_{L}(l, \ldots) \frac{1}{P^{2}} \mathcal{A}_{R}(-l, \ldots) \delta^{(4)}\left(P_{L}+P_{R}\right)
$$

where $P_{L}$ and $P_{R}$ are the sum of the external momenta on the left and on the right of the diagrams, with $P=-P_{L}=P_{R}$, and $l:=\lambda \tilde{\lambda}$. We have also explicitly written a delta function for momentum conservation, which we recast as $\int d^{4} P \delta^{(4)}\left(P_{L}+P\right) \delta^{(4)}\left(P_{R}-P\right)$.

We wish to associate each delta function with the corresponding amplitude on the left or on the right of the diagram. We also expand $P$ as in (2.14), so that the result is

$$
\int d^{4} P \delta^{(4)}\left(P_{L}+l+z \eta\right) \mathcal{A}_{L}(l, \ldots) \frac{1}{P^{2}} \delta^{(4)}\left(-P_{R}+l+z \eta\right) \mathcal{A}_{R}(-l, \ldots) .
$$

Now, consider each sub-amplitude, for example the one on the left which, together with its delta function, reads

$$
\mathcal{A}_{L}(l, \ldots) \delta^{(4)}\left(P_{L}+l+z \eta\right)
$$

The delta function can be interpreted as imposing the condition that the sum of the external momenta is now $P_{L}+z \eta$, rather than $P_{L}$. Analogously, on the right this sum will be $P_{R}-z \eta$. Overall momentum is trivially conserved, but at the level of each subamplitude we have to shift the sum of the external momenta by $\pm z \eta$.

One way to do this is to imagine that the extra momentum is used entirely to shift the momentum of a single particle on the left, say $p_{k}$, by $z \eta, p_{k} \rightarrow p_{k}(z):=p_{k}+z \eta$, and the momentum of a single particle on the right, $p_{l}$, by $-z \eta, p_{l} \rightarrow p_{l}(z):=p_{l}-z \eta$. Imposing the requirement that the new momenta are still null leads to $\eta=\lambda_{k} \tilde{\lambda}_{l}$ or $\eta=\lambda_{l} \tilde{\lambda}_{k}$, as discussed before. One could alternatively attribute the momentum shifts to more than one particle, which would lead to the more generic shifts (2.6).

\section{Application to MHV gravity amplitudes}

In the following we will compute the MHV scattering amplitude $\mathcal{M}\left(1^{-}, 2^{-}, 3^{+}, \ldots, n^{+}\right)$ for $n$ gravitons. We will choose the two negative helicity gravitons $1^{-}$and $2^{-}$as reference legs. This is a particularly convenient choice, as it reduces the number of terms arising in the recursion relation to a minimum. The shifts for the momenta of particles 1 and 2 are

$$
p_{1} \rightarrow p_{1}+z \lambda_{2} \tilde{\lambda}_{1}, \quad p_{2} \rightarrow p_{2}-z \lambda_{2} \tilde{\lambda}_{1} .
$$

In terms of spinors, the shifts are realised as

$$
\lambda_{1} \rightarrow \hat{\lambda}_{1}:=\lambda_{1}+z \lambda_{2}, \quad \tilde{\lambda}_{2} \rightarrow \hat{\tilde{\lambda}}_{2}:=\tilde{\lambda}_{2}-z \tilde{\lambda}_{1}
$$




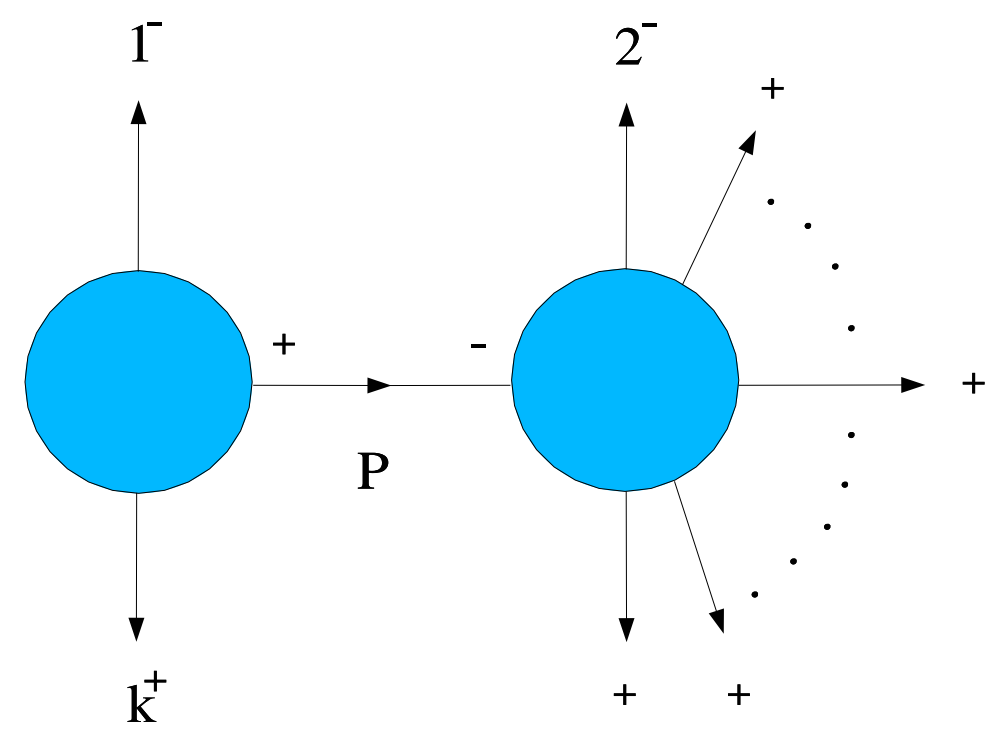

Figure 1: One of the terms contributing to the recursion relation for the MHV amplitude $\mathcal{M}\left(1^{-}, 2^{-}, 3^{+}, \ldots, n^{+}\right)$. The gravity scattering amplitude on the right is symmetric under the exchange of gravitons of the same helicity. In the recursion relation, we sum over all possible values of $k$, i.e. $k=3, \ldots, n$. This amounts to summing over cyclical permutations of $(3, \ldots, n)$.

with $\lambda_{2}$ and $\tilde{\lambda}_{1}$ unmodified.

Let us consider the possible recursion diagrams that can arise. There are only two possibilities, corresponding to the two possible internal helicity assignments, $(+-)$ and $(-+)$ :

1. The amplitude on the left is googly $(++-)$, whereas on the right there is an MHV gravity amplitude with $n-1$ legs (see Figure 1).

2. The amplitude on the right is googly, and the amplitude on the left is MHV (see Figure 2).

We recall that a gravity amplitude is symmetric under the interchange of identical helicity gravitons; this implies that we have to sum $n-2$ diagrams for each of the configurations in Figures 1 and 2. Each diagram is then completely specified by choosing $k$, with $k=$ $3, \ldots, n$.

However, it is easy to see that diagrams of the type $\mathbf{2}$. actually give a vanishing 


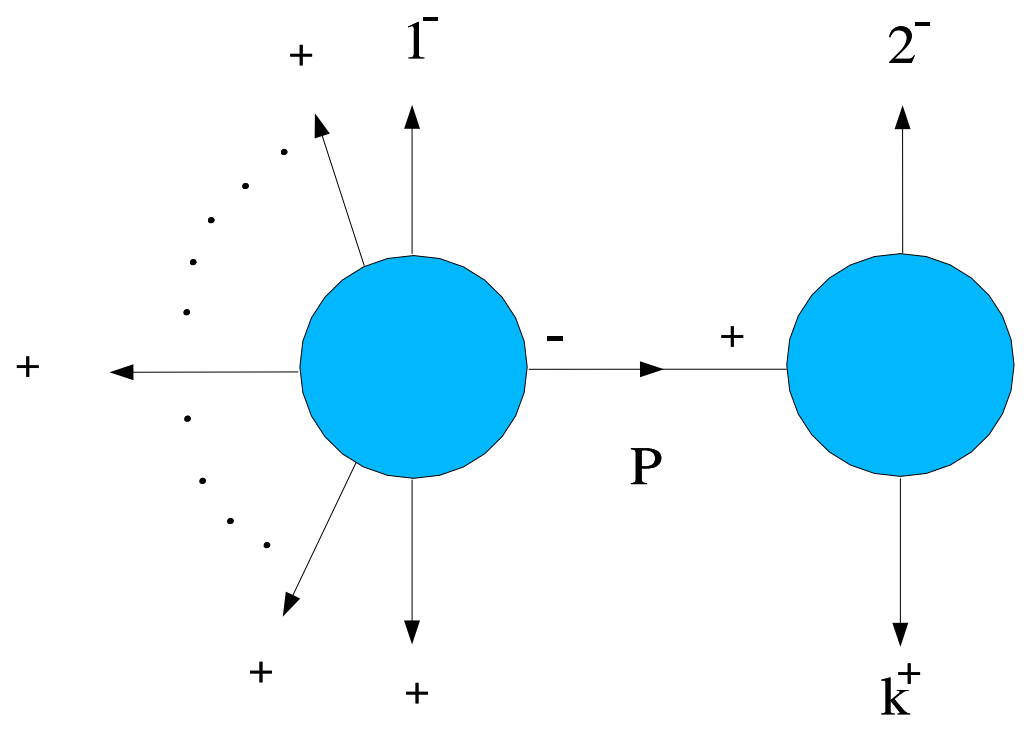

Figure 2: This class of diagrams also contributes to the recursion relation for the $M H V$ amplitude $\mathcal{M}\left(1^{-}, 2^{-}, 3^{+}, \ldots, n^{+}\right)$; however, each of these diagrams vanishes if the shifts (3.2) are performed.

contribution. Indeed, they are proportional to

$$
[k \hat{P}]=\frac{[k|\hat{P}| \hat{2}\rangle}{\langle\hat{P} \hat{2}\rangle}=\frac{[k|P| 2\rangle}{\langle\hat{P} \hat{2}\rangle}=0,
$$

where the last equality follows from $P=p_{k}+p_{2}$. Hence we will have to compute diagrams of type 1. only. We will do this in the following.

\subsection{Four, five and six graviton scattering}

To show explicitly how our recursion relation generates amplitudes, we will now derive the 4,5 and 6 point MHV scattering amplitudes.

We start with the four point case. There are two diagrams to sum, one of which is represented in Figure 3; the other is obtained by swapping the labels 4 with 3. For the diagram in Figure 3, we have

$$
\mathcal{M}^{(4)}=\mathcal{M}_{L} \frac{1}{P^{2}} \mathcal{M}_{R}
$$




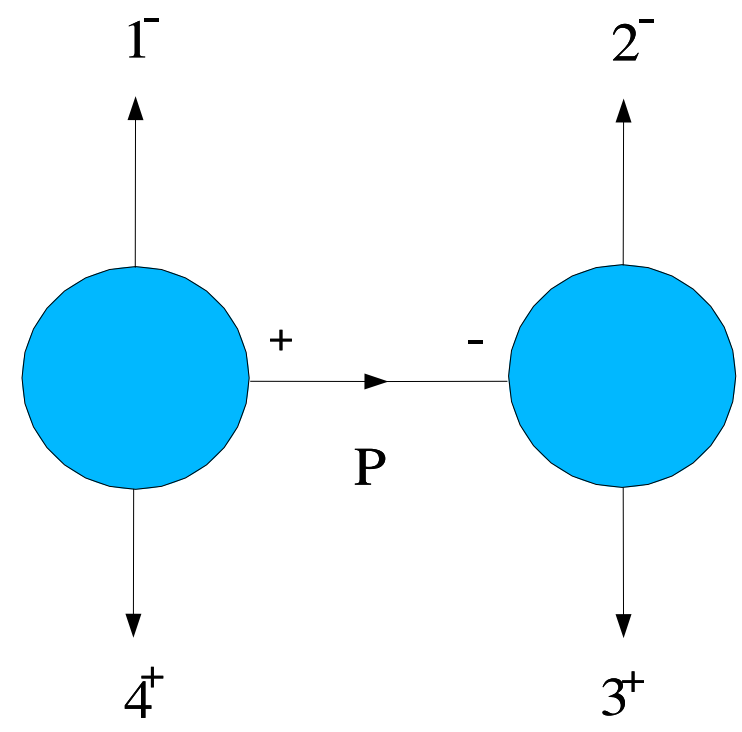

Figure 3: One of the two diagrams contributing to the recursion relation for the $M H V$ amplitude $\mathcal{M}\left(1^{-}, 2^{-}, 3^{+}, 4^{+}\right)$. The other is obtained from this by cyclically permuting the labels $(3,4)$ - i.e. swapping 3 with 4.

where the superscript denotes the label on the positive helicity leg in the trivalent vertex,

$$
\begin{aligned}
\mathcal{M}_{L} & =\left(\frac{[\hat{P} 4]^{3}}{[41][1 \hat{P}]}\right)^{2}, \\
\mathcal{M}_{R} & =\left(\frac{\langle\hat{P} 2\rangle^{3}}{\langle 23\rangle\langle 3 \hat{P}\rangle}\right)^{2},
\end{aligned}
$$

and $P^{2}=\left(p_{1}+p_{4}\right)^{2}$. Using

$$
\langle i \hat{P}\rangle=\frac{\langle i|P| 1]}{[\hat{P} 1]}
$$

we find, after a little algebra,

$$
\mathcal{M}^{(4)}=\frac{\langle 12\rangle^{6}[14]}{\langle 14\rangle\langle 23\rangle^{2}\langle 34\rangle^{2}} .
$$

The full amplitude is $\mathcal{M}\left(1^{-}, 2^{-}, 3^{+}, 4^{+}\right)=\mathcal{M}^{(3)}+\mathcal{M}^{(4)}$. Thus, we conclude that the four point MHV amplitude generated by our recursion relation is given by

$$
\mathcal{M}\left(1^{-}, 2^{-}, 3^{+}, 4^{+}\right)=\frac{\langle 12\rangle^{6}[14]}{\langle 14\rangle\langle 23\rangle^{2}\langle 34\rangle^{2}}+3 \leftrightarrow 4 .
$$


It is easy to check that this agrees with the conventional formula for this amplitude

$$
\mathcal{M}\left(1^{-}, 2^{-}, 3^{+}, 4^{+}\right)=\frac{\langle 12\rangle^{8}[12]}{N(4)\langle 34\rangle}
$$

where

$$
N(n):=\prod_{1 \leq i<j \leq n}\langle i j\rangle
$$

or, equivalently, with the expression derived from the appropriate KLT relation, Eq. (A.2). For the five graviton scattering case, our recursion relation yields a sum of three diagrams. A calculation similar to that illustrated previously for the four-point case leads to the result

$$
\mathcal{M}\left(1^{-}, 2^{-}, 3^{+}, 4^{+}, 5^{+}\right)=\frac{\langle 12\rangle^{6}[15][34]}{\langle 15\rangle\langle 23\rangle\langle 24\rangle\langle 34\rangle\langle 35\rangle\langle 45\rangle}+\mathcal{P}^{\mathrm{c}}(3,4,5),
$$

where $\mathcal{P}^{\mathrm{c}}(3,4,5)$ means that we have to sum over cyclic permutations of the labels $3,4,5$. The conventional formula for the five graviton MHV scattering amplitude is

$$
\mathcal{M}\left(1^{-}, 2^{-}, 3^{+}, 4^{+}, 5^{+}\right)=\frac{\langle 12\rangle^{8}}{N(5)}([12][34]\langle 13\rangle\langle 24\rangle-[13][24]\langle 12\rangle\langle 34\rangle) .
$$

Using standard spinor identities and momentum conservation, it is straightforward to check that our expression (3.11) agrees with this (alternatively, one can use the KLT relation (A.3) $)$.

For the six graviton scattering amplitude, our recursion relation yields a sum of four terms,

$$
\begin{aligned}
\mathcal{M}\left(1^{-}, 2^{-}, 3^{+}, 4^{+}, 5^{+}, 6^{+}\right)= & \frac{\langle 12\rangle^{6}[16]}{\langle 16\rangle} \cdot \frac{1}{\langle 26\rangle\langle 34\rangle\langle 35\rangle\langle 45\rangle} \\
& \left(\frac{[34]}{\langle 23\rangle\langle 24\rangle} \frac{\langle 2|3+4| 5]}{\langle 56\rangle}+\frac{[45]}{\langle 24\rangle\langle 25\rangle} \frac{\langle 2|4+5| 3]}{\langle 36\rangle}+\frac{[53]}{\langle 23\rangle\langle 25\rangle} \frac{\langle 2|5+3| 4]}{\langle 46\rangle}\right) \\
+ & \mathcal{P}^{\mathrm{c}}(3,4,5,6) .
\end{aligned}
$$

The known formula for this amplitude is

$\mathcal{M}\left(1^{-}, 2^{-}, 3^{+}, 4^{+}, 5^{+}, 6^{+}\right)=\langle 12\rangle^{8}\left(\frac{[12][45][3|4+5| 6\rangle}{\langle 15\rangle\langle 16\rangle\langle 12\rangle\langle 23\rangle\langle 26\rangle\langle 34\rangle\langle 36\rangle\langle 45\rangle\langle 46\rangle\langle 56\rangle}+\mathcal{P}(2,3,4)\right)$,

where $\mathcal{P}(2,3,4)$ indicates permutations of the labels $2,3,4$. We have checked numerically that the formula (3.13) agrees with this expression.

\subsection{General formula for MHV scattering}

Recursion relations of the form given in [19], or the graviton recursion relation given here, naturally produce general formulae for scattering amplitudes. For a suitable choice 
of reference spinors, these new formulae can often be simpler than previously known examples. For the choice of reference spinors 1,2, which we have made above, the graviton recursion relation is particularly simple, as it produces only one term at each step. This immediately suggests that one can use it to generate an explicit expression for the $n$-point amplitude. This turns out to be the case, and experience with the use of our recursion relation leads us to propose the following new general formula for the $n$-graviton $\mathrm{MHV}$ scattering amplitude. This is (labels 1,2 carry negative helicity, the remainder carry positive helicity)

$$
\mathcal{M}\left(1,2, i_{1}, \cdots, i_{n-2}\right)=\frac{\langle 12\rangle^{6}\left[1 i_{n-2}\right]}{\left\langle 1 i_{n-2}\right\rangle} G\left(i_{1}, i_{2}, i_{3}\right) \prod_{s=3}^{n-3} \frac{\left\langle 2\left|i_{1}+\ldots+i_{s-1}\right| i_{s}\right]}{\left\langle i_{s} i_{s+1}\right\rangle\left\langle 2 i_{s+1}\right\rangle}+\mathcal{P}\left(i_{1}, \ldots, i_{n-2}\right),
$$

where

$$
G\left(i_{1}, i_{2}, i_{3}\right)=\frac{1}{2} \frac{\left[i_{1} i_{2}\right]}{\left\langle 2 i_{1}\right\rangle\left\langle 2 i_{2}\right\rangle\left\langle i_{1} i_{2}\right\rangle\left\langle i_{2} i_{3}\right\rangle\left\langle i_{1} i_{3}\right\rangle} .
$$

(For $n=5$ the product term is dropped from (3.15)). It is straightforward to check that this amplitude satisfies the recursion relation with the choice of reference legs $1^{-}$and $2^{-}$.

The known general MHV amplitude for two negative helicity gravitons, 1 and 2, and the remaining $n-2$ with positive helicity, is given by [23]

$$
\mathcal{M}(1,2,3, \cdots, n)=\langle 12\rangle^{8}\left[\frac{[12][n-2 n-1]}{\langle 1 n-1\rangle} \frac{1}{N(n)} \prod_{i=1}^{n-3} \prod_{j=i+2}^{n-1}\langle i j\rangle F+\mathcal{P}(2, \ldots, n-2)\right]
$$

where

$$
F= \begin{cases}\prod_{l=3}^{n-3}\left[l\left|\left(p_{l+1}+p_{l+2}+\cdots+p_{n-1}\right)\right| n\right\rangle & n \geq 6 \\ 1 & n=5\end{cases}
$$

We have checked numerically, up to $n=11$, that our formula (3.15) gives the same results as (3.17).

\section{Applications to other field theories}

One of the striking features of the $\mathrm{BCFW}$ proof of the $\mathrm{BCF}$ recursion relations is that it is almost not needed to specify the theory with which we are dealing. Indeed, in [20] the only step where specifying the theory did matter was in the estimate of the behaviour of the scattering amplitudes $\mathcal{M}(z)$ as $z \rightarrow \infty$, which was important to assess the possible existence of boundary terms in the recursion relation. This leads us to conjecture that recursion relations could be a more generic feature of massless (or spontaneously broken) field theories in four dimensions. ${ }^{5}$ After all, the BCF recursion relations, as well as the

\footnotetext{
${ }^{5}$ This was also suggested in [21].
} 
recursion relation for gravity amplitudes discussed in this paper, just reconstruct a treelevel amplitude (which is a rational function) from its poles.

Let us focus on massless $\lambda\left(\phi^{\dagger} \phi\right)^{2}$ theory in four dimensions. We use the spinor helicity formalism, meaning that each momentum will be written as $p_{a \dot{a}}=\lambda_{a} \tilde{\lambda}_{\dot{a}}$. A scalar propagator $1 / P^{2}$ connects states of opposite "helicity", which here just means that the propagator is $\left\langle\phi(x) \phi^{\dagger}(0)\right\rangle$, with $\langle\phi(x) \phi(0)\rangle=\left\langle\phi^{\dagger}(x) \phi^{\dagger}(0)\right\rangle=0$. Now consider a Feynman diagram contributing to an $n$-particle scattering amplitude, and let us shift the momenta of particles $k$ and $l$ as in (2.3). As for the Yang-Mills case discussed in [20], there is a unique path of propagators going from particle $k$ to particle $l$. Each of these propagators contributes $1 / z$ at large $z$, whereas vertices are independent of $z$. We thus expect Feynman diagrams contributing to the amplitude to vanish in the large- $z$ limit.

An exception to the above reasoning is represented by those Feynman diagrams where the shifted legs belong to the same vertex; these diagrams are $z$-independent, and hence not suppressed as $z \rightarrow \infty$. In order to deal with this problematic situation, and ensure that the full amplitude computed from Feynman diagrams $\mathcal{M}(z)$ vanishes as $z \rightarrow \infty$ we propose two alternatives.

Firstly, if one considers $\left(\phi \phi^{\dagger}\right)^{2}$ theory without any group structure, one can remove the problem by performing multiple shifts. This possibility has already been used in the context of the rational part of one-loop amplitudes in pure Yang-Mills [21]. In our case, it is sufficient to shift at least four external momenta.

Alternatively, we can consider $\left(\phi \phi^{\dagger}\right)^{2}$ theory with global symmetry group $U(N)$ and $\phi$ in the adjoint. In this case we can group the amplitude into colour-ordered partial amplitudes, as in the Yang-Mills case. Then, for any colour-ordered amplitude one can always find a choice of shifts such that the shifted legs do not belong to the same Feynman vertex. The procedure can be repeated for any colour ordering, and the complete amplitude is obtained by summing over non-cyclic permutations of the external legs.

In this way, the appearance of a boundary term $\mathcal{C}_{\infty}$ can be avoided, and one can thus derive a recursion relation for scattering amplitudes similar to (2.10). A similar analysis can be carried out in other theories, possibly in the presence of spontaneous symmetry breaking, etc. We expect this to play an important rôle in future studies.

\section{Acknowledgements}

It is a pleasure to thank Valya Khoze, Marco Matone and Sanjaye Ramgoolam for discussions. GT acknowledges the support of PPARC. 


\section{Appendix A: KLT relations}

For completeness, in this appendix we write the field theory limit of the KLT relations [42] for the case of four, five and six points:

$$
\begin{aligned}
\mathcal{M}(1,2,3) & =-i \mathcal{A}(1,2,3) \mathcal{A}(1,2,3) \\
\mathcal{M}(1,2,3,4) & =-i s_{12} \mathcal{A}(1,2,3,4) \mathcal{A}(1,2,4,3) \\
\mathcal{M}(1,2,3,4,5) & =i s_{12} s_{34} \mathcal{A}(1,2,3,4,5) \mathcal{A}(2,1,4,3,5) \\
& +i s_{13} s_{24} \mathcal{A}(1,3,2,4,5) \mathcal{A}(3,1,4,2,5) \\
\mathcal{M}(1,2,3,4,5,6) & =-i s_{12} s_{45} \mathcal{A}(1,2,3,4,5,6)\left[s_{35} \mathcal{A}(2,1,5,3,4,6)\right. \\
& \left.+\left(s_{34}+s_{35}\right) \mathcal{A}(2,1,5,4,3,6)\right] \\
& +\mathcal{P}(2,3,4) .
\end{aligned}
$$

In these formulae, $\mathcal{M}(\mathcal{A})$ denotes a tree-level gravity (Yang-Mills colour-ordered) amplitude, $s_{i j}:=\left(p_{i}+p_{j}\right)^{2}$, and $\mathcal{P}(2,3,4)$ stands for permutations of $(2,3,4)$. The relation for a generic number of particles can be found in [43]. 


\section{References}

[1] For a survey of the latest developments, see the web-page of the London Mathematical Society Workshop on Twistor String Theory, Oxford 10-14 January 2005, http://www.maths.ox.ac.uk/ lmason/Tws/

[2] E. Witten, Perturbative gauge theory as a string theory in twistor space, Commun. Math. Phys. 252, 189 (2004) hep-th/0312171.

[3] F. Cachazo, P. Svrček and E. Witten, MHV vertices and tree amplitudes in gauge theory, JHEP 0409 (2004) 006, hep-th/0403047.

[4] A. Brandhuber, B. Spence and G. Travaglini, One-Loop Gauge Theory Amplitudes in N=4 super Yang-Mills from MHV Vertices, Nucl. Phys. B 706 (2005) 150, hep-th/0407214.

[5] Z. Bern, L. J. Dixon, D. C. Dunbar and D. A. Kosower, Fusing gauge theory tree amplitudes into loop amplitudes, Nucl. Phys. B 435 (1995) 59, hep-ph/9409265.

[6] Z. Bern, L. J. Dixon, D. C. Dunbar and D. A. Kosower, One Loop N Point Gauge Theory Amplitudes, Unitarity And Collinear Limits, Nucl. Phys. B 425 (1994) 217, hep-ph/9403226.

[7] Z. Bern, V. Del Duca, L. J. Dixon, and D. A. Kosower, All Non-MaximallyHelicity-Violating One-Loop Seven-Gluon Amplitudes in N=4 Super Yang-Mills Theory, hep-th/0410224.

[8] C. Quigley and M. Rozali, One-Loop MHV Amplitudes in Supersymmetric Gauge Theories, hep-th/0410278.

[9] J. Bedford, A. Brandhuber, B. Spence and G. Travaglini, A Twistor Approach to One-Loop Amplitudes in $\mathcal{N}=1$ Supersymmetric Yang-Mills Theory, Nucl. Phys. B 706 (2005) 100, hep-th/0410280

[10] L. J. Dixon, E. W. N. Glover and V. V. Khoze, MHV Rules for Higgs Plus MultiGluon Amplitudes, JHEP 0412 (2004) 015, hep-th/0411092.

[11] J. Bedford, A. Brandhuber, B. Spence and G. Travaglini, Non-supersymmetric loop amplitudes and MHV vertices, Nucl. Phys. B 712 (2005) 59, hep-th/0412108.

[12] F. Cachazo, P. Svrček and E. Witten, Gauge Theory Amplitudes In Twistor Space And Holomorphic Anomaly, JHEP 0410 (2004) 077, hep-th/0409245.

[13] F. Cachazo, Holomorphic Anomaly Of Unitarity Cuts And One-Loop Gauge Theory Amplitudes, hep-th/0410077.

[14] R. Britto, F. Cachazo, B. Feng, Computing One-Loop Amplitudes from the Holomorphic Anomaly of Unitary Cuts, hep-th/0410179. 
[15] S. J. Bidder, N. E. J. Bjerrum-Bohr, L. J. Dixon and D. C. Dunbar, $N=1$ Supersymmetric One-loop Amplitudes and the Holomorphic Anomaly of Unitarity Cuts, hep-th/0410296.

[16] R. Britto, F. Cachazo and B. Feng, Generalized unitarity and one-loop amplitudes in $N=4$ super-Yang-Mills, hep-th/0412103.

[17] Z. Bern, L. J. Dixon and D. A. Kosower, All Next-to-Maximally-Helicity-Violating One-Loop Gluon Amplitudes in N=4 Super-Yang-Mills Theory, hep-th/0412210.

[18] R. Roiban, M. Spradlin and A. Volovich, Dissolving N=4 loop amplitudes into $Q C D$ tree amplitudes, hep-th/0412265.

[19] R. Britto, F. Cachazo and B. Feng, New recursion relations for tree amplitudes of gluons, hep-th/0412308.

[20] R. Britto, F. Cachazo, B. Feng and E. Witten, Direct proof of tree-level recursion relation in Yang-Mills theory, hep-th/0501052.

[21] Z. Bern, L. J. Dixon and D. A. Kosower, On-shell recurrence relations for one-loop QCD amplitudes, hep-th/0501240.

[22] S. Weinberg, The Quantum theory of fields. Vol. 1: Foundations, Cambridge University Press, 1995.

[23] F. A. Berends, W. T. Giele and H. Kuijf, On Relations Between Multi - Gluon And Multigraviton Scattering, Phys. Lett. B 211, 91 (1988).

[24] S. Giombi, R. Ricci, D. Robles-Llana and D. Trancanelli, A note on twistor gravity amplitudes, JHEP 0407 (2004) 059, hep-th/0405086.

[25] J-B. Wu and C-J Zhu, MHV Vertices and Scattering Amplitudes in Gauge Theory, JHEP 0407 (2004) 032 hep-th/0406085.

[26] V. P. Nair, A note on MHV amplitudes for gravitons, hep-th/0501143.

[27] Z. Bern, N. E. J. Bjerrum-Bohr and D. C. Dunbar, Inherited twistor-space structure of gravity loop amplitudes, hep-th/0501137.

[28] L. J. Dixon, Calculating scattering amplitudes efficiently, hep-ph/9601359.

[29] C. J. Zhu, The googly amplitudes in gauge theory, JHEP 0404 (2004) 032, hep-th/0403115.

[30] G. Georgiou and V. V. Khoze, Tree amplitudes in gauge theory as scalar MHV diagrams, JHEP 0405 (2004) 070, hep-th/0404072.

[31] J-B. Wu and C-J Zhu, MHV Vertices and Fermionic Scattering Amplitudes in Gauge Theory with Quarks and Gluinos, hep-th/0406146. 
[32] I. Bena, Z. Bern and D. A. Kosower, Twistor-space recursive formulation of gauge theory amplitudes, hep-th/0406133.

[33] D. Kosower, Next-to-Maximal Helicity Violating Amplitudes in Gauge Theory, hep-th/0406175.

[34] F. Cachazo, P. Svrček and E. Witten, Twistor space structure of one loop amplitudes in gauge theory, JHEP 0410 (2004) 074, hep-th/0406177.

[35] G. Georgiou, E. W. N. Glover and V. V. Khoze, Non-MHV Tree Amplitudes in Gauge Theory, JHEP 0407, 048 (2004), hep-th/0407027.

[36] V.V. Khoze, Gauge Theory Amplitudes, Scalar Graphs and Twistor Space, To appear in From Fields to Strings: Circumnavigating Theoretical Physics, in memory of Ian Kogan, hep-th/0408233.

[37] I. Bena, Z. Bern, D. A. Kosower and R. Roiban, Loops in Twistor Space, hep-th/0410054

[38] R. Britto, F. Cachazo, B. Feng, Coplanarity in Twistor Space of $N=4$ Next-ToMHV One-Loop Amplitude Coefficients, hep-th/0411107.

[39] S. J. Bidder, N. E. J. Bjerrum-Bohr, D. C. Dunbar and W. B. Perkins, Twistor Space Structure of the Box Coefficients of $N=1$ One-loop Amplitudes, hep-th/0412023.

[40] Z. Bern, D. Forde, D. A. Kosower and P. Mastrolia, Twistor-inspired construction of electroweak vector boson currents, hep-ph/0412167.

[41] Z. Bern, Perturbative quantum gravity and its relation to gauge theory, Living Rev. Rel. 5, 5 (2002) gr-qc/0206071.

[42] H. Kawai, D. C. Lewellen and S. H. H. Tye, A Relation Between Tree Amplitudes Of Closed And Open Strings, Nucl. Phys. B 269 (1986) 1.

[43] Z. Bern, L. J. Dixon, M. Perelstein and J. S. Rozowsky, Multi-leg one-loop gravity amplitudes from gauge theory, Nucl. Phys. B 546 (1999) 423, hep-th/9811140. 